\title{
Search for Low-Mass Planets Around Late-M Dwarfs Using IRD
}

\section{Masashi Omiya ${ }^{1}$, Bun'ei Sato ${ }^{1}$, Hiroki Harakawa ${ }^{1}$, Masayuki} Kuzuhara $^{2}$, Teruyuki Hirano ${ }^{2}$, Norio Narita ${ }^{3}$ and IRD team

\author{
${ }^{1}$ Tokyo Institute of Technology, 2-12-1 Ookayama, Meguro-ku, Tokyo 152-8551, Japan email: \\ omiya.m@geo.titech.ac.jp \\ ${ }^{2}$ The University of Tokyo, Tokyo 113-0033, Japan \\ ${ }^{3}$ National Astronomical Observatory of Japan, 2-21-1 Osawa, Mitaka, Tokyo 181-8588, Japan
}

\begin{abstract}
We have a plan to conduct a Doppler planet search for low-mass planets around nearby middle-to-late $\mathrm{M}$ dwarfs using IRD. IRD is the near-infrared high-precision radial velocity instrument for the Subaru 8.2-m telescope. We expect to achieve the accuracy of the radial velocity measurements of $1 \mathrm{~m} / \mathrm{s}$ using IRD with a frequency comb as a wavelengh calibrator. Thus, we would detect super-Earths in habitable zone and low-mass rocky planets in closein orbits around late-M dwarfs. In this survey, we aim to understand and discuss statistical properties of low-mass planets around low-mass $M$ dwarfs compared with those derived from theoretical simulations.
\end{abstract}

Keywords. stars: planetary systems, stars: low-mass, brown dwarfs, techniques: radial velocities,instrumentation: spectrographs

\section{Introduction}

The stellar radial velocity (RV) techniques have uncovered more than 500 extrasolar planets around various types of stars and the planets show remarkable diversity in their properties so far (e.g.Udry \& Santos 2007). However, planets around lower-mass stars, such as M- and L-type dwarfs, have not been well harvested yet, because they are intrinsically faint in optical wavelength region even using telescopes with large aperture, like Subaru. Low-mass end of the current samples of planet searches is limited to about M3-type dwarfs with a mass of around $0.3 \mathrm{M}_{\odot}$. The lower luminosity of these stars brings the potentially habitable zone closer to the star, making planets in shorter-period orbits more interesting for the search of life outside our own solar system.

A habitable Earth-mass planet around a $0.1 \mathrm{M}_{\odot}$ star can impart 20 times larger velocity variations of the host star, compared to the velocity amplitude of the Sun caused by the Earth $\left(\sim 10 \mathrm{~cm} \mathrm{~s}^{-1}\right)$, which makes it easier to detect the planet. Planets in such short orbits are much more favorable for RV measurements and have a much higher chance for transiting their host star (e.g., Charbonneau et al. 2009). Recently, Bonfils et al. (2013) obtained $\eta_{\oplus}=0.41_{-0.13}^{+0.54}$, the frequency of habitable planets orbiting $\mathrm{M}$ dwarfs $(1 \leqslant$ $\left.m \sin i \leqslant 10 \mathrm{M}_{\oplus}\right)$. Although the uncertainties are fairly large due to the limited number of samples and measurement precision for faint lower mass stars, the results encourage us to search for habitable planets around such M dwarfs more intensively.

\section{Near-Infrared Doppler Instrament for the Subaru telescope (IRD)}

To realize planet searches around lower mass stars, a near-infrared spectroscopy is necessary, because the flux distribution of late-M dwarfs has the peak in infrared wavelength 
regions and they are very faint in the visual. RVs measured from nIR spectra also likely offer the advantage that activity induced "jitter" is reduced relative to measurements in the visible because of the smaller contrast between the cool inhomogeneities caused by activity and the average stellar surface at the longer wavelengths.

In order to make the detection of Earth-like exoplanets possible by the Subaru telescope, we are currently developing an infrared RV spectrometer system known as IRD (Tamura et al. 2012). IRD would achive a high precision velocity measurements comparable to the current optical Doppler instruments $\left(\sim 1 \mathrm{~m} \mathrm{~s}^{-1}\right)$ using an original frequency comb (i.e. Astro-comb, Li et al. 2008) for the wavelength calibration. IRD is designed to cover wavelengths from 1.0 to $1.75 \mathrm{um}$ with a 70,000 spectral resolution and, has a foreoptics part for star-light and laser frequency comb injection into fibers, and a backend spectrometer optics and detector unit.

We estimated achievable RV precision for a slowly rotating late-M star using a theoretical spectrum. Based on the current design, IRD is planned to have a wavelength resolution of 70,000 by 3 pixel sampling and covers $\mathrm{J}$ and $\mathrm{H}$ bands. With these setting, expected RV precision per exposure for J-band is $4 \mathrm{~m} \mathrm{~s}^{-1}$ and for $\mathrm{H}$-band is $1 \mathrm{~m} \mathrm{~s}^{-1}$ when we assume 100,000 photons per pixel for the continuum level. If we combine the two bands, expected RV precision should be $0.9 \mathrm{~m} \mathrm{~s}^{-1}$.

\section{An infrared RV survey of late-M dwarfs}

We are planning to carry out a near-Infrared RV survey of nearby middle-to-late M dwarfs to search for low-mass planets using IRD. The aims of the survey are to detect super-Earths in habitable zone and low-mass rocky planets in close-in orbits around lowmass stars $\left(0.1-0.4 M_{\odot}\right)$ and to understand properties of low-mass planets around such stars.

Low mass M dwarf stars as samples of our RV survey are selected from catalogs of M-dwarfs (e.g. Lepine \& Gaidos 2011) following criteria: absolute magnitude $M_{V}<11$ to select $\mathrm{M}$ dwarf stars with masses of less than $0.4 M_{\odot}, J$-band magnitude $J<10$ to obtain data with high signal-to-noise ratio in a short exposure time. From the list of stars extracted from the catalogs, we should choose about 300 suitable stars for a Doppler survey using stellar activity indicators and number of stars with similar mass and multiplicity based on initial spectroscopic observation.

We performed simulations of observations in order to estimate detectability of various planets depending on the number, interval, and duration of observations. Stellar mass, stellar jitter, and RV measurement error is assumed to be $0.3 M_{\odot}, 1 \mathrm{~m} \mathrm{~s}^{-1}$, and $1 \mathrm{~m} \mathrm{~s}^{-1}$, respectively, which means we assume "quiet" stars for this simulation. Minimum detectable mass of planets becomes larger if stellar jitter becomes larger. At the beginning of the survey, "quick look" observations are effective to find candidates of short-period planets. Only a few consecutive measurements in a few days enables us to identify stars showing large RV variations, which are candidates harboring short-period $\left(\lesssim 10\right.$ d) Neptune- and Jupiter-class planets $\left(\gtrsim 10 M_{\oplus}\right)$, and Jupiter- class ones in habitable zone $(P \sim 10-50 d)$. To derive occurrence rate of planets by setting constraints on parameters for planet formation theories, more extensive observations are necessary not only for planet-harboring stars but also those without detectable planets in order to uniform detectability of planets. In this case, we can identify most of the planets more massive than $20 M_{\oplus}$ within orbital period of $\sim 100$ days including habitable zone, and discuss their statistical properties compared with those derived from theoretical simulations. In order to detect rocky planets, more observations are required. Figure 1 shows detectability of planets when we conduct 3 consecutive-nights observations every month 


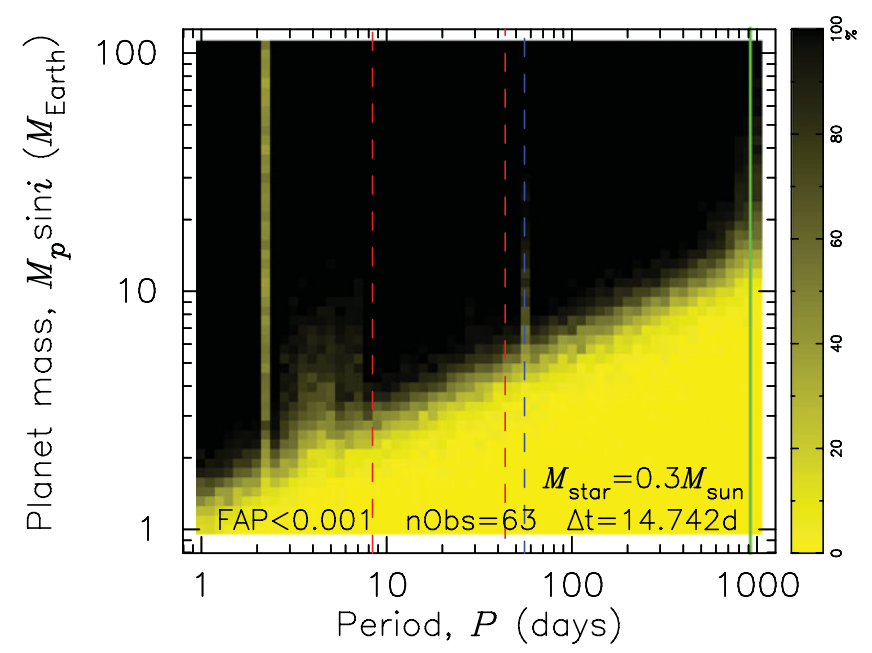

Figure 1. Detectability of planets for "intensive observations". Observations with 3 consecutive nights every month for 3 years are assumed. The total number of data points is about 60 . Red lines indicate inner and outer edges of habitable zone around a $0.3 M_{\odot}$ star (Selsis et al. 2007). Blue line indicates a snow line around the star derived by Ida \& Lin (2005). Green line indicates the observation period of the simulation. In this observing mode, the detection limit can be pushed down to $1-2 M_{\oplus}$ at $P \sim 1 \mathrm{~d}$ around a $0.3 M_{\odot}$ star.

for 3 years. The detection limit could be pushed down to $3 M_{\oplus}$ at $P \sim 10 \mathrm{~d}$ and $7 M_{\oplus}$ at $P \sim 100 \mathrm{~d}$ for 3 -years observations. In this case, we could detect Earth-mass planets at $P \sim 1 \mathrm{~d}$.

\section{Acknowledgements}

We are grateful to the IRD team and SEEDS project member for their inputs to the science and instrumentation of IRD. We are grateful to the AO development team. We also thank the Subaru telescope team for their telescope/instrument supports. This research is supported by Ministry of Education, Culture, Sports, Science and Technology of Japan, Grant-in-Aid for Specially Promoted Research.

\section{References}

Bonfils, X., Delfosse, X., Udry, S., Forveille, T., Mayor, M., Perrier, C., Bouchy, F., Gillon, M., Lovis, C., Pepe, F., Queloz, D., Santos, N. C., Ségransan, D., \& Bertaux, J.-L., 2013 A\&A, 549 , id.A109

Charbonneau, D., Berta, Z. K., Irwin, J., Burke, C. J., Nutzman, P., Buchhave, L. A., Lovis, C., Bonfils, X., Latham, D. W., Udry, S., Murray-Clay, R. A., Holman, M. J., Falco, E. E., Winn, J. N., Queloz, D., Pepe, F., Mayor, M., Delfosse, X., \& Forveille, T. 2009 Nature, 462,891

Ida, S. \& Lin, D. N. C. $2005 A p J, 626,1045$

Lépine, S. \& Gaidos, E. 2011 AJ, 142, 138L

Li, C.-H., Benedick, A. J., Fendel, P., Glenday, A. G., Kärtner, F. X., Phillips, D. F., Sasselov, D., Szentgyorgyi, A., \& Walsworth, R. L. 2008 Nature, 452, 610

Selsis, F., Kasting, J. F., Levrard, B., Paillet, J., Ribas, I., \& Delfosse, X. 2007 A $\mathscr{E} A, 476,1373$

Tamura, M. et al. 2012 SPIE, 8446, article id. 84461T

Udry, S. \& Santos, N. C. 2007 ARA\&SA, 45, 397 